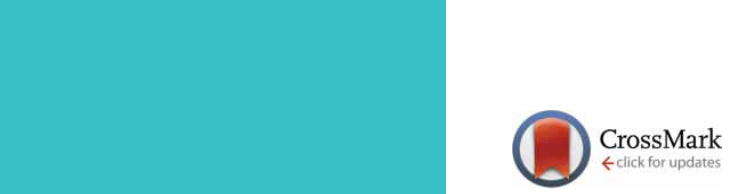

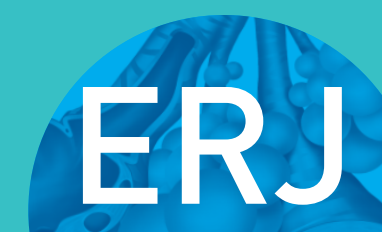

open research

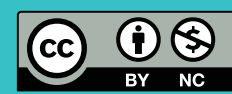

\section{ERS Presidential Summit 2018: multimorbidities and the ageing population}

\author{
Mina Gaga (1) ${ }^{1}$, Pippa Powell ${ }^{2}$, Marta Almagro ${ }^{3}$, Ioanna Tsiligianni $\mathbb{1}^{4}$, \\ Stelios Loukides ${ }^{5}$, Josep Roca ${ }^{6}$, Matthew Cullen ${ }^{2}$, Anita K. Simonds ${ }^{7}$, \\ Brian Ward ${ }^{8}$, Isabel Saraiva ${ }^{2}$, Thierry Troosters ${ }^{9}$ and Carlos Robalo Cordeiro ${ }^{10}$
}

Affiliations: ${ }^{1} 7$ th Resp. Med. Dept and Asthma Center, Athens, Greece. ${ }^{2}$ European Lung Foundation, Sheffield, UK. ${ }^{3}$ ELF Bronchiectasis Patient Advisory Committee, Sheffield, UK. ${ }^{4}$ University of Crete, Crete, Greece. ${ }^{5}$ University of Athens Medical School, Athens, Greece. ${ }^{6}$ University of Barcelona, Barcelona, Spain. ${ }^{7}$ Royal Brompton Hospital and Imperial College, London, UK. ${ }^{8}$ European Respiratory Society, Brussels, Belgium. ${ }^{9}$ Katholieke Universiteit Leuven, Leuven, Belgium. ${ }^{10}$ Coimbra University Hospital, Coimbra, Portugal.

Correspondence: M. Gaga is the European Respiratory Society (ERS) Past President, I. Tsiligianni is the International Primary Care Respiratory Group President, T. Troosters is the ERS President Elect and C. Robalo Cordeiro is the ERS Secretary General.

ABSTRACT As the average age of the population increases, so will the prevalence of chronic respiratory diseases and associated multimorbidity. This will result in a more complex clinical environment. Part of the solution will be to allow patients to be co-creators in the design of their care. It will also require clinicians to shift in their current approaches to care, step out of the disease- or pathology-oriented approach and embrace new ideas. In an effort to prepare the respiratory community for the challenge, we reflect on concepts to empower patients via multidisciplinary systems, new technologies and transition from end-of-life care to advanced care planning.

@ERSpublications

Increasing life expectancy will lead to the need for better respiratory and multimorbid care http://bit.ly/2KgLvGu

Cite this article as: Gaga M, Powell P, Almagro M, et al. ERS Presidential Summit 2018: multimorbidities and the ageing population. ERJ Open Res 2019; 5: 00126-2019 [https://doi.org/ 10.1183/23120541.00126-2019].

Received: 21 May 2019 | Accepted after revision: 29 July 2019

Copyright $\odot$ ERS 2019. This article is open access and distributed under the terms of the Creative Commons Attribution Non-Commercial Licence 4.0. 


\section{Introduction}

In 2017, the European Respiratory Society (ERS) started work on a new strategy looking forward to 2025. As part of that strategy, ERS launched a survey asking all its members and key stakeholders to identify emerging trends that may affect ERS in the future. The survey ran from December 2017 to January 2018. Within the circulation list were 200 patient organisations from the European Lung Foundation (ELF) patient organisation network, from whom 32 responses were received (16\% response rate).

One of the key issues highlighted by patients and patient organisations was the challenge of an ageing population. This is in keeping with the further increase in life expectancy and increase in mean population age that has been predicted [1], and is also outlined in the editorial by GAGA [2] published in this issue of ERJ Open Research.

As the average age of the population increases, so does the prevalence of chronic respiratory diseases and their health impact. In line with these changes, the number of people that will suffer from respiratory diseases will increase in the next decades. McNeE et al. [3] predicted that there would be a significant increase in chronic pulmonary disease over the next four decades, due to increases in the world's population aged $>60$ years, and the link between ageing and chronic inflammatory disease. This has become even more complicated as most people with any long-term condition have multiple conditions [4]. Moreover, medication adherence falls as the number of diseases and medications increase [5].

In addition, (elderly) patients with chronic respiratory diseases are likely to display complex disease. Figure 1, from an article by Jones et al. [6], demonstrates the issue of comorbidities well, showing those presented by patients with COPD. That paper also highlights the opportunity of improved screening for COPD if people with comorbidities would be screened and diagnosed earlier.

Another interesting study showed a higher prevalence of most of the diseases, as comorbid conditions, in elderly (>65 years) people living with COPD compared with non-COPD subjects. Analysis of the temporal order of disease diagnoses showed that comorbid conditions in elderly patients with COPD tend to appear after the diagnosis of the obstructive disease, rather than before it, indicating a further need for awareness [7].

This multimorbidity pattern makes daily clinical practice complicated, especially as there is a vertical disease-oriented approach and mono-disease guidelines.

Since 1) patients with chronic respiratory disease are likely to be living with more than one condition and 2) their functional problems may not be due to their respiratory pathology alone, a session dedicated to how an ageing population with more chronic conditions will need to be treated by a multidisciplinary team, how technology could improve care, and how to address palliative and end-of-life issues was timely at the ERS Presidential Summit in Athens, Greece, in June 2018.

\section{The International Classification of Functioning, Disability and Health}

In 2001, the World Health Organization (WHO) introduced the International Classification for Functioning, Disability and Health (ICF), replacing the Classification of Impairment, Disability and Handicap [8]. Although the model (figure 2) can be criticised [9], the ICF is particularly useful when

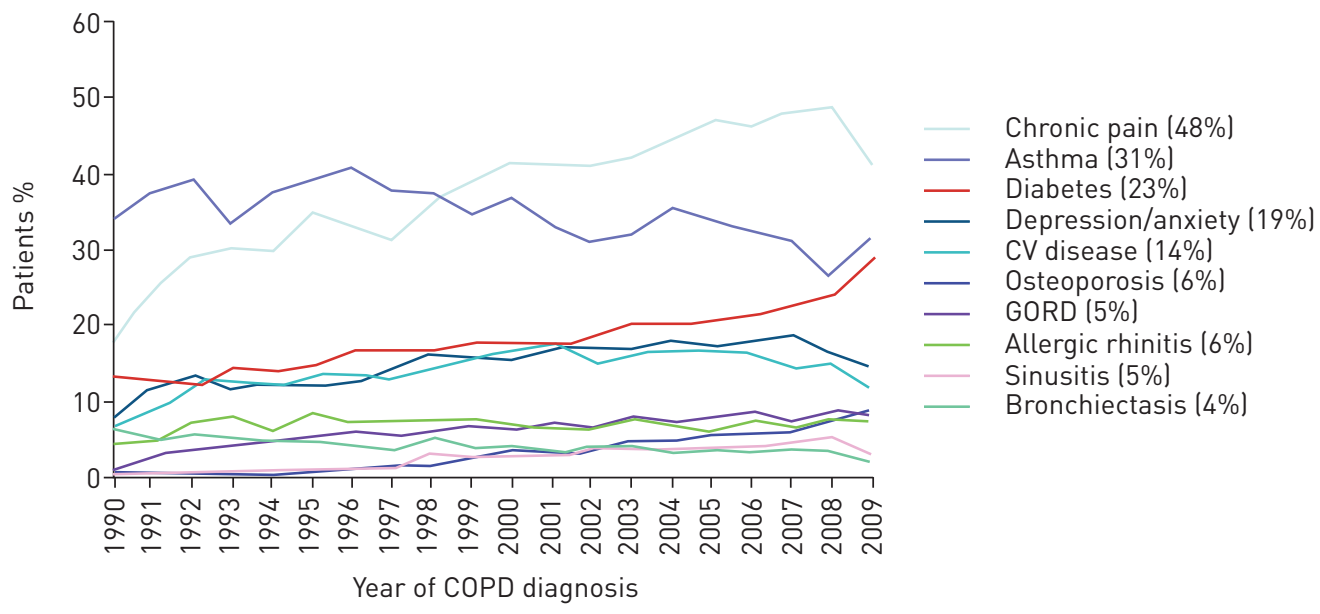

FIGURE 1 Proportion of patients with COPD who had other comorbidities. Values in parentheses are 2007 prevalence. CV: cardiovascular; GORD: gastro-oesophageal reflux disease. Reproduced and modified from [6] with permission. 


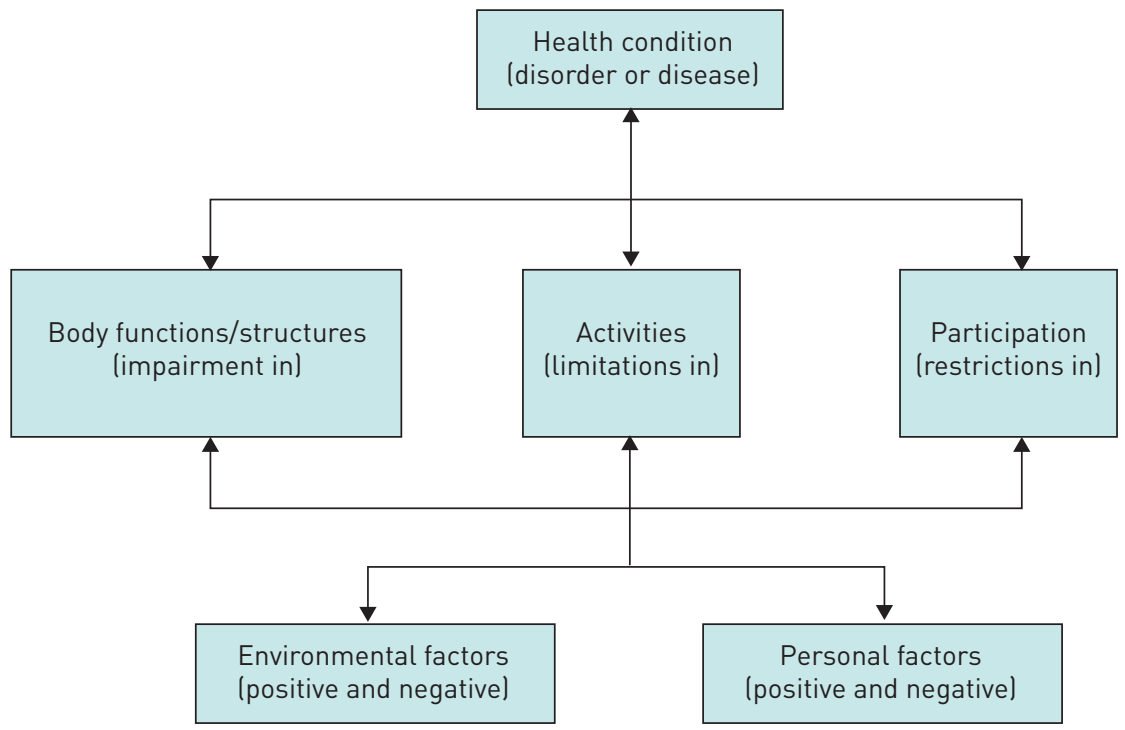

FIGURE 2 International Classification for Functioning, Disability and Health concept as proposed by the World Health Organization in 2001. Reproduced and modified from [8], and published under a Creative Commons Attribution Non-Commercial ShareAlike 3.0 licence.

dealing with patients in need of multidisciplinary interventions. It classifies body functions, activities and participation in daily life, rather than diseases or their severity (as in the International Classification of Diseases). Through a patient-centred (rather than single disease-centred) approach, body functions, activities and level of participation can be mapped out, and strategies to enhance function and participation can be agreed for individual patients. In a respiratory patient, body functions are not only lung function, but also muscle function and mental function, for example. Activities are things patients do in their daily life (e.g. walking on the level or climbing one flight of stairs), and participation reflects the meaningful interaction of patients with family members, work-related aspects of their disease, etc. Importantly, the model also considers environmental and social factors as elements that determine the level of function and participation of an individual. For example, a patient with more social support will typically have a higher level of participation in a given activity limitation. Patients that have aids (e.g. a wheeled walker) will be able to have a better level of activity for a given level of bodily function. While core sets of outcomes to assess bodily function, activity and participation are available for several chronic diseases, including lung disease [10], it is merely the use of the ICF concept that helps clinicians step out of the disease- or pathology-oriented approach and reflect on the broader needs of a patient. It also helps encourage discussion of the desired levels of activity and participation, regardless of the underlying primary disease.

\section{The role of multidisciplinary teams}

A multidisciplinary approach has been described as: "When professionals from a range of disciplines work together to deliver comprehensive care that addresses as many of the patient's needs as possible. This can be delivered by a range of professionals functioning as a team under one organizational umbrella or by professionals from a range of organizations, including private practice, brought together as a unique team. As a patient's condition changes over time, the composition of the team may change to reflect the changing clinical and psychosocial needs of the patient" [11].

A multidisciplinary team should include:

- the patient

- the general practitioner (GP)

- the specialist(s) and the subspecialities (e.g. pulmonary plus rheumatology or oncology)

- other (allied) healthcare professionals (e.g. practice nurse, pharmacist, nutritionist, mental health expert, physiotherapist, speech and language therapist, occupational therapist and social workers)

Multidisciplinary teams convey many benefits to both patients and the healthcare professionals working collaboratively on the team. These include improved health outcomes and enhanced satisfaction for patients, and the more efficient use of resources and enhanced job satisfaction for healthcare professionals. In order to achieve optimum functioning of the team and effective patient outcomes, the roles of the multidisciplinary team members in care planning and delivery must be clearly negotiated and defined. 
The role of the patient

The patient is a key part of the multidisciplinary team, and can bring innovative ideas and viewpoints to improve the process.

From a practical perspective, a multidisciplinary team is a huge advantage for the patient as it should make their life easier. It should result in a holistic and customised treatment experience, which should allow the patient to be educated and empowered to self-manage their condition, and have better overall health and quality of life. It can also have the added benefit of making research easier.

In an example given by Marta Almagro, a patient with bronchiectasis who presented at the ERS summit in Athens, it is important to have the bronchiectasis specialist working closely with the infectious diseases team with regards treatment for cross interaction and using less antibiotics in the long term:

We are a whole person - we are not just a respiratory disease or a digestive system. Our job as a patient is to take care of ourselves. For me, being treated by a multidisciplinary team means that I can be empowered, I can self-manage and I have an improved quality of life.

\section{The role of the GP}

The GP or family physician is the first point of contact for most patients, providing open and unlimited access, dealing with all health problems and interacting with them over their life course, and therefore should be an anchor of the multidisciplinary team [12].

The GP should make efficient use of healthcare resources by coordinating care, working with other professionals in the primary care setting and managing the interface with other specialities, taking an advocacy role for the patient when needed. All GPs should develop a person-centred approach, orientated to the individual, the patient's family and their community; promote patient empowerment; and be responsible for the provision of longitudinal continuity of care, as determined by the needs of the patient.

The GP has a specific decision-making process determined by the prevalence and incidence of illness in the community, and manages both acute and chronic health problems of individual patients. The GP also promotes health and wellbeing both by appropriate and effective intervention, with a specific responsibility for the health of the community.

In the field of pulmonary health, the GP can be involved in many areas of care including prevention, diagnosis and treatment of chronic airways diseases, infections and tuberculosis. There should also be easy access for referral to hospital or community-based specialists, and to pulmonary rehabilitation and palliative and end-of-life care.

The Alma-Ata Declaration of 1978 identified primary care as the cornerstone of healthy, thriving communities; the foundation for integrating the full spectrum of health and social services to improve health outcomes; and the key to sustainable, accessible and equitable health systems. 40 years later, in October 2018, the Astana declaration, which was unanimously endorsed by all WHO Member States, made pledges in four key areas: 1) make bold political choices for health across all sectors; 2) build sustainable primary healthcare; 3) empower individuals and communities; and 4) align stakeholder support to national policies, strategies and plans [13].Transmural interactions, where the GP is and feels supported and respected by the multidisciplinary team, contribute to the functionality of such teams. Information from the "home" setting is often crucial in decisions on the best or most feasible care trajectory. Healthcare systems should invest in the integration of primary care as a full member of transmural multidisciplinary teams for complex disease management.

\section{The role of the specialist}

A specialty in medicine is a branch of medical practice. After completing medical school, physicians or surgeons usually further their medical education in a specific specialty of medicine by completing a multiple-year residency to become a medical specialist. As many respiratory patients will have more than one chronic condition, they could be under the care of more than one specialist. Coordination between different specialists is vitally important, and can help find indicators and patterns between diseases.

In order for a multidisciplinary team to be effective, the specialist must respect and trust the competencies of other team members. The approach that should be taken is based on protocols, but more importantly on interactions between all the team members.

\section{The role of nurses and other allied health professionals}

In the context of a multidisciplinary team, it is common to have a respiratory nurse as the first direct contact - acting as one point of contact and giving better disease-related education. They can play a key role in educating patients about a healthy lifestyle. 
Depending on the underlying problem and the observed comorbidity, other healthcare professionals have key roles to play in the diagnostic track, in treating the patient or even in supervising the management of patients. Table 1 gives an overview of healthcare professionals and their typical roles, as well as some specific disease conditions where they play a key role in diagnosis and treatment.

Mental health is a key issue not only when a patient is diagnosed but also throughout the life course of disease and especially if a patient has a life-limiting condition. Therefore, mental health support is a key area that may need to be covered. Rehabilitation is important in several chronic diseases. Often, the functional limitations of patients and their participation in daily life is not primarily limited by their lung problems but rather by weakness, lack of stamina, fatigue, anxiety, etc. Treatment of the underlying lung condition will therefore fail to maximally restore participation of patients. Multidisciplinary rehabilitation has therefore been successfully implemented for most chronic lung conditions, including lung cancer, COPD, cystic fibrosis, interstitial lung disease, pulmonary hypertension and bronchiectasis [14].

\section{Current issues and best practices}

The concept of a multidisciplinary team with all the stakeholders listed in this paper working well together is the ideal scenario. However, there are many challenges that can prevent this from being effective. These challenges include interprofessional differences in the management of the patient and regional differences in competency profiles. These can be an issue when guidelines for various diseases need to be integrated and implemented. Time constraints, work load and inadequate patient selection can often prevent professionals from completing and reading the multidisciplinary integrated care pathway, and goal setting has been regarded by some as time consuming. Although disciplines and countries can learn from one another, there are many different healthcare systems across Europe as well as a resistance to change from professionals and patients.

Education in this area is very important and there is an expanding body of literature looking at interprofessional teamwork. Fox et al. [15] recently carried out a scoping review to explore interprofessional education that was designed to teach and/or assess interprofessional teamwork skills to students from two or more different health professions. The main finding of the review was that, regardless of the teaching method that was used, educational activities where interprofessional teams interact produce positive changes in student perceptions and attitudes towards multidisciplinary teamwork and practice.

In Italy, the national respiratory society developed a model for COPD management that could work as an example for other chronic respiratory conditions [16]. This approach was developed during a face-to-face meeting where the issues were divided into training, organisation, responsibilities and sustainability. The areas that were highlighted as needing work included improved specialist training by better integration of universities and teaching hospitals, and better organisation between specialists and GPs in the local community. One of the key issues highlighted was that the sustainability of the whole system was dependent on clear diagnostic-therapeutic pathways developed by GPs and specialists, with buy-in of other healthcare professionals, that all stakeholders could follow and feed into.

\section{The role of digital technology, including patient experiences}

There have been some examples of multidisciplinary team approaches in respiratory care. One study in the UK used a multidisciplinary team for COPD decisions and used telehealth as an assessment tool. This study demonstrated a $90 \%$ success rate in offering lower-cost advice and education for oxygen treatment, and referral to rehabilitation for the treatment of comorbidities. There was bidirectional learning between

\section{TABLE 1 Roles within the multidisciplinary team}

\begin{tabular}{lc} 
Health professional & Role and/or disease condition \\
\hline $\begin{array}{l}\text { Physiotherapist } \\
\text { Nutritional specialist }\end{array}$ & Airway secretion problems, rehabilitation \\
Occupational therapist & Cystic fibrosis, obesity, malnourishment \\
Speech and language therapist & Frailty, home adaptations \\
$\begin{array}{l}\text { Psychologist } \\
\text { Pain management expert }\end{array}$ & Swallowing, dysphagia \\
Smoking cessation expert & Mental health \\
Sleep technician/expert & Elderly, cancer, all aspects of pain control \\
Microbiologist & All patients with lung disease who continue to smoke \\
\end{tabular}


the specialist and the GP [17]. Telehealth integrated asthma and COPD services, which are well developed in countries such as the Netherlands, that establish a clear collaboration between GPs and specialists could be adapted and implemented in other countries as well [18].

In another study, however, family practitioners and internists disagreed or were neutral to self-managing solitary pulmonary nodules, which is a major issue for patients at high risk of lung cancer. In this case, there was a lack of education, lack of collaboration, loss to follow-up and improper assessment, resulting in nonbeneficial effects on patient outcomes [19].

One of the best overall examples of how multidisciplinary teams can work comes from the Finnish asthma and COPD programmes: 10-year programmes to improve the care of asthma and COPD in which a multidisciplinary team approach was used and the coordinator of care was the GP. In the case of asthma [20], the main action focussed on the fact that asthma needs treatment with anti-inflammatory agents (inhaled corticosteroids). The programme resulted in a decrease in the rate of exacerbations and the number of hospitals days, and an increase in the prescription rate of inhaled corticosteroids. There was an overall higher awareness about asthma. The same was seen in the COPD programme, which focussed on education about the quality of spirometry, training to improve calibration in spirometry and education about smoking cessation. The programme resulted in an increased diagnostic rate of COPD, a decreased rate of exacerbations and hospitalisations, a decrease in smoking rates and decreased costs of the disease [21].

Digital technology is booming and can potentially support patients. Recently a systematic review identified $>100$ apps for patients with respiratory and allergic diseases. $80 \%$ of the apps were developed by medical technology companies and $18 \%$ by medical doctors. Apps provide self-monitoring, patient feedback and education leading to increased patient empowerment [22]. Digital technology in general is believed to enhance patient self-management if they fit three conditions: 1) the technology should help to empower patients; 2) the technology should contribute to information about the patient; and 3) the technology should fit cost effectively into services. Enthusiasm around technology-based interventions has been constrained by negative clinical trials, predominantly of telemedicine, also applied in patients with respiratory diseases $[23,24]$. During the ERS Presidential Summit, however, a positive example was given from NextCare. This programme, developed in Spain, focuses on conditions most commonly seen alongside pulmonary disease, such as cardiovascular disease, type II diabetes, lung cancer, anxiety and depression. Among other areas, the programme explores the potential of technology to support collaborative work fostering interactions within multidisciplinary teams [25].

The programme has a patient-centred approach aiming to: 1) help predict health risk and service selection [26];2) facilitate self-management and promotion of a healthy lifestyle, including rehabilitation [27, 28]; 3) aid in the management of complex chronic conditions [25]; 4) help with the transfer of diagnostic tests from specialist care to primary care (spirometry [29]); and 5) enhance technology support for the services being deployed in the programme.

The programme therefore fits nicely with the 5P approach for mobile health proposed by GoRINI et al. [30] emphasising: 1) support for predictive intervention; 2) personalisation; 3) involvement of patients in preventive programmes; 4) social features (communication); and 5) a user-centred design (psychocognitive). The main goal of the NextCare project is to bring together information from the healthcare system to help the patient to manage their condition and facilitate collaborative work between patients and professionals (including primary care, specialist care and social care). Examples of the data used to input into the patient's health records include patient-reported outcomes, social support received and information from sensors that the patients may use.

A recent article published in BMJ Open [26], as part of the NextCare programme, analyses the potential of improving care for individuals with COPD when comorbidities are considered. In the study, the health risk distribution of $>264000$ patients with COPD was compared with a similar-age population in Catalonia and to the whole population of the region. It is of note that registry data showed high potential for predication of mortality, hospitalisations and use of healthcare resources. The report emphasises the high potential of bringing together clinical data and population-based data, taking into account multimorbidity, for enhanced clinical risk prediction and service selection.

Overall, the results currently generated in NextCare encourage use of digital technologies to: 1) support patient empowerment by using the personal health folder as a self-management tool; 2) include collaborative adaptive case management into the clinical records; 3) develop enhanced multilevel predictive modelling to support clinical decision making; and 4) further explore the potential of cloud-based computing for management of health data.

A simple practical example of how digital tools can work was given by Matt Cullen (an IPF patient from Ireland, also living with rheumatoid arthritis) during the ERS Presidential Summit. Matt volunteered to 
take part in the use of an app (patientMpower, Dublin, Ireland) for patients with idiopathic pulmonary fibrosis for a 6-week trial period. However, he continued to use the app after the trial period was over, as he felt empowered. He had found that the app enabled him to have more control in managing his health condition.

Benefits both now and into the future include: allowing the user to record objective measurements such as forced vital capacity using a spirometer; allowing the user to record subjective information about their condition and how they feel in a journal; patients being able to use the app in a way that suits their individual needs; and recording information over time to help patients to be aware of changes to their symptoms, which can lead to more informed discussions with healthcare provider and can help aid research.

While such systems may not be beneficial when rolled out in large and general populations of patients, Matt clearly benefitted from the intervention. It provided him with more confidence about the management of his disease and his healthcare team with objective data about his health. How such systems should (not) be reimbursed and how medical teams should be involved practically in looking at the stream of data potentially flowing in their direction should be further studied.

\section{Advanced care planning, and palliative and end-of-life care}

Palliative care is "An essential component of comprehensive care for people with complex chronic or acute, life-threatening, or life-limiting health conditions that should be practiced by all healthcare and social care providers and by palliative care specialists, and that can be provided in any health-care setting, including patients' own homes". Palliative care should provide multicomponent care, including physiological, social and spiritual support. This can be needed for a significant part of the care of chronically ill patients and is shown in figure 3 [31]. What is key is that palliative care does manage symptoms but it can also involve active interventions.

It is also very important to note that palliative care is broader than end-of-life care - in fact, end-of-life care is only one very specific part of palliative care (figure 4) [32]. It is this unclear differentiation that can cause concern and apprehension when discussing these issues.

The need for palliative and end-of-life care will affect every one of us. Worldwide, there are 56.2 million deaths per year, $45 \%$ of which involve serious health-related suffering. Global access to palliative care is poor in low-income countries and is not optimal in more developed countries. The aim of palliative care is to improve quality of life, symptoms and comfort, and it should involve patients and caregivers, and end-of-life planning. Advanced care planning should take place early in the disease process so that time is available for discussion with family members and the healthcare team. Advanced care planning also should allow room for reconsideration and adjustment. Advanced care planning can be offered in

Categories of serious health-related suffering

$\begin{aligned} & \text { Health conditions } \\ & \text { or stage of disease }\end{aligned}-\begin{aligned} & \text { Chronic or acute life- } \\ & \text { threatening or life-limiting } \\ & \text { disease, ill health, and injury } \\ & \text { Not associated with a life- } \\ & \text { threatening or life-limiting } \\ & \text { health condition }\end{aligned}$

Palliative care health-related interventions

Palliative care interventions primarily outside of healthcare

Health conditions that generate serious health-related suffering mitigated by other health and social interventions, and preferably not managed by palliative care, yet often are in improverished settings 
FIGURE 4 The part that end-of-life care plays in palliative care.

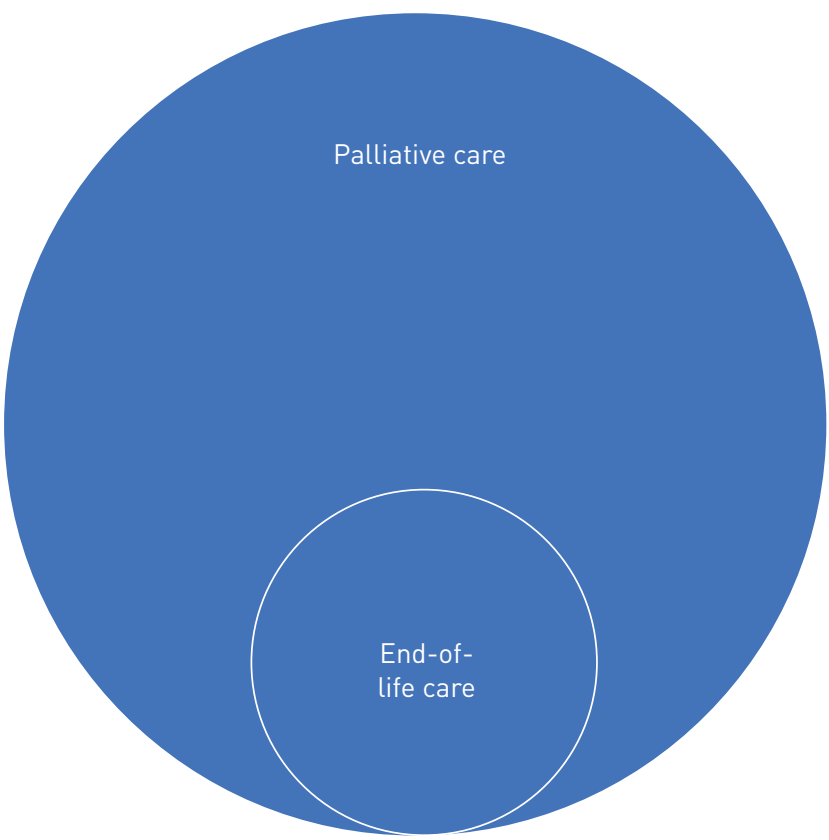

nurse-led programmes and improve the end-of-life communication between the healthcare team and the patient [33].

Oncology palliative care is often well managed with recognised pathways. However, there is consistent evidence that palliative medicine is less well developed and delivered in chronic respiratory and multimorbid conditions.

Integrating palliative medicine and respiratory medicine will enable respiratory teams to deliver highly effective palliative care; and it is therefore essential to educate the multidisciplinary team and have the right people on board.

A randomised controlled trial was performed that included patients with COPD, interstitial lung disease, lung cancer and heart failure. One group was given an intervention to manage breathlessness involving physiotherapy and drugs. The other half were randomised to standard routine care. The primary outcome measure for the trial was mastery of breathlessness - how "in control" of their breathlessness the participants felt.

The trial saw not only significant improvements in the primary outcome measure but also trends in improvement in secondary measures. One unexpected outcome was an increase in survival rate in all disease areas except lung cancer. One explanation for this finding is that it is likely that providing good palliative medicine is a marker for good general care [34].

In another study by SMALLWOOD et al. [35], an integrated approach was used in a mixed group with advanced lung disease. Individuals were provided with a plan for managing their symptoms. The study found that although respiratory admissions did not change, there were significant reductions in emergency admissions, therefore suggesting that patients had more control of their symptoms and were having fewer exacerbations. The result was that by engaging patients, more took part in planning their care and only $24-25 \%$ of the patients in this study died in hospital as compared to $70 \%$ in usual care. The large majority of patients in this study died at home.

A focus group study in Norway looked at the impact of end-of-life care planning for people with life-threatening pulmonary disease. In that study, the investigators used an advance care planning tool and found that using it increased patients' feeling of "comforting safety" [36].

There are a number of barriers to effective palliative care. Patients can have the view that they want to concentrate on staying alive, they may not be clear who will look after them when they are sick, and they may be confused about the difference between palliative and end-of-life care. For physicians, they may feel that they do not have the time to hold the discussion with the patient and they may have concerns that the patient is not ready to discuss the issue. What often occurs is that conversations occur at time of acute exacerbation, which can often be stressful. 
The solution is that palliative care should be integrated from the time of diagnosis, as show in figure 5 [37].

What role for ERS/ELF?

Multimorbidity and multidisciplinary teams

- ERS could feature good practice models for multidisciplinary care and provide guidance as to issues such as when specialist care is needed.

- Early diagnosis is key and ERS should collaborate with other disciplines to ensure that respiratory disease is picked up as quickly as possible.

- Cost-effective interventions should not be forgotten in ERS guidelines and activities (pulmonary rehabilitation and smoking cessation).

- Sharing information is important - what is the most effective way to do it and how can ERS/ELF help with that? For example, ERS could help to identify what minimal respiratory information should be shared on electronic platforms. This could help harmonise platforms across the globe.

- ELF could help with needs assessment and user validation of apps developed by ERS members for respiratory patients. It could help in making such apps available in several languages.

\section{Ageing and palliative care}

- Should terminology be changed: end-of-life to advanced care planning? And it is important to emphasise this is one part of the palliative care spectrum.

- ERS/ELF should be promoting healthy lung ageing from before birth.

- ERS should investigate innovative partnerships (postmen etc.) to reach the elderly with lung disease who are isolated.

- ERS should work to include the topic in education in medical schools and in ERS educational programmes.

- ERS should work with national societies, primary care and patient organisations to raise awareness about the important of advance care planning in lung disease.

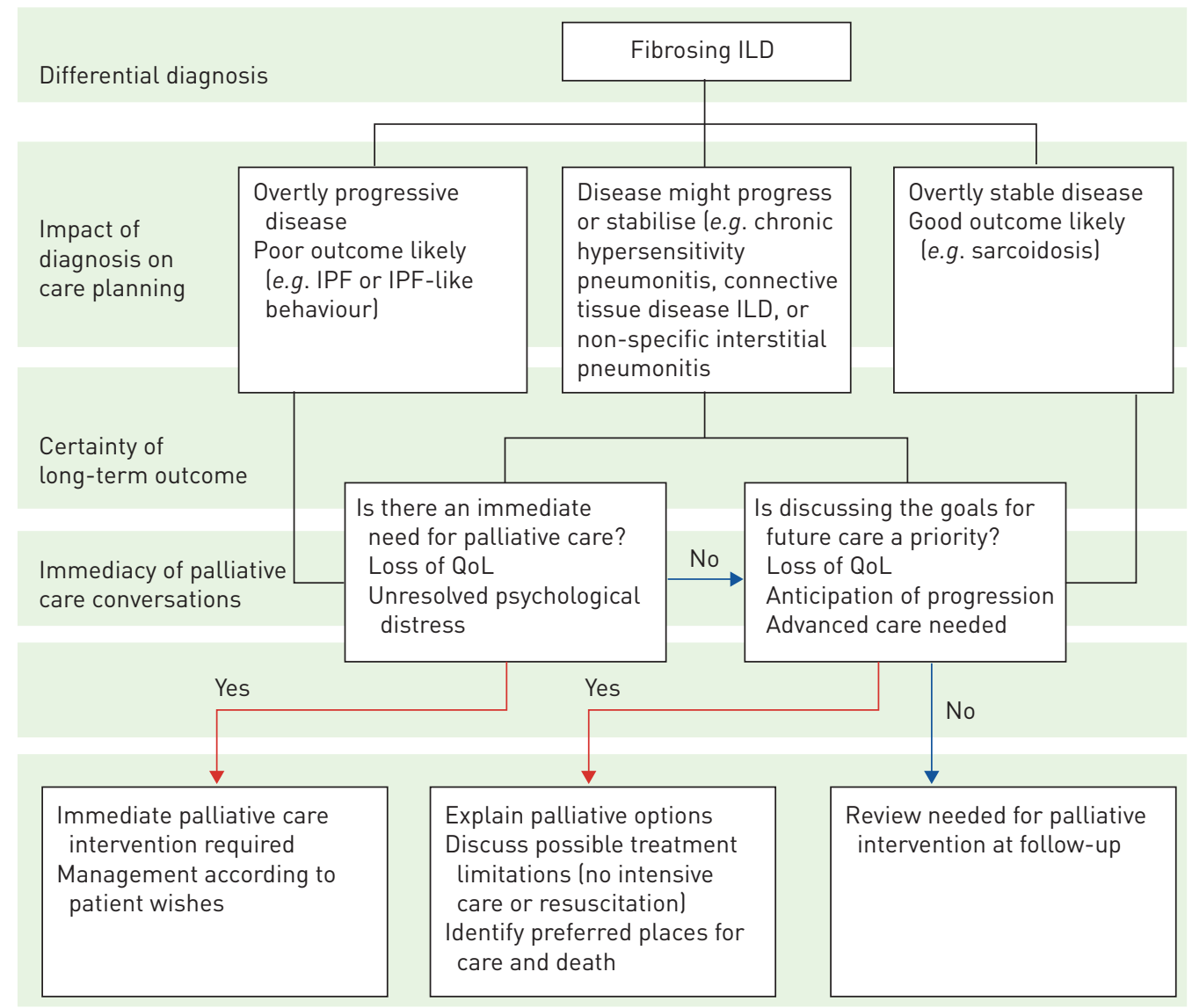

FIGURE 5 The integration of palliative care in idiopathic pulmonary fibrosis (IPF). ILD: interstitial lung disease; QoL: quality of life. Reproduced and modified from [37] with permission from the publisher. 
- ERS should look at the timing of the discussion of advance care discussion and advise lung health experts of when the best opportunities may be to introduce discussions and prompt patients to initiative the discussions as well.

Conflict of interest: M. Gaga has received departmental grants and honoraria from AZ, GSK, Menarini, Chiesi, Novartis, BMS, MSD, Roche, Elpen and Pharmaten outside of this work. P. Powell is an employee of the European Lung Foundation. M. Almagro reports nonfinancial support from Polyphor outside the submitted work. I. Tsiligianni reports personal fees from Novartis, grants and personal fees from GSK, and personal fees and other support from Boehringer Ingelheim, outside the submitted work. Dr. Loukides has nothing to disclose. Dr. Roca has nothing to disclose. M. Cullen has nothing to disclose. A.K. Simonds has nothing to disclose. B. Ward is an employee of the European Respiratory Society. I. Saraiva has nothing to disclose. T. Troosters has nothing to disclose. C. Robalo Cordeiro has nothing to disclose.

\section{References}

1 Foreman KJ, Marquez N, Dolgert A, et al. Forecasting life expectancy, years of life lost, and all-cause and cause-specific mortality for 250 causes of death: reference and alternative scenarios for 2016-40 for 195 countries and territories. Lancet 2018; 392: 2052-2090.

2 Gaga M. ERS Presidential Summit: redesigning the future of patient care. ERJ Open Res 2019; 5: 00139-2018.

3 MacNee W, Rabinovich RA, Choudhury G. Ageing and the border between health and disease. Eur Respir J 2014; 44: 1332-1352.

4 Barnett K, Mercer SW, Norbury M, et al. Epidemiology of multimorbidity and implications for health care, research, and medical education: a cross-sectional study. Lancet 2012; 380: 37-43.

5 Gellad WF, Grenard JL, Marcum ZA. A systematic review of barriers to medication adherence in the elderly: looking beyond cost and regimen complexity. Am J Geriatr Pharmacother 2011; 9: 11-23.

6 Jones RC, Price D, Ryan D, et al. Opportunities to diagnose chronic obstructive pulmonary disease in routine care in the UK: a retrospective study of a clinical cohort. Lancet Respir Med 2014; 2: 267-276.

7 Tényi Á, Vela E, Cano I, et al. Risk and temporal order of disease diagnosis of comorbidities in patients with COPD: a population health perspective. BMJ Open Respir Res 2018; 5: e000302.

8 WHO. ICF: International Classification of Functioning, Disability and Health. Geneva, World Health Organization, 2001.

9 Heerkens YF, de Weerd M, Huber M, et al. Reconsideration of the scheme of the international classification of functioning, disability and health: incentives from the Netherlands for a global debate. Disabil Rehabil 2017; 40: 603-611.

10 Marques A, Jácome C, Gonçalves A, et al. Validation of the Comprehensive ICF Core Set for obstructive pulmonary diseases from the patient's perspective. Int J Rehabil Res 2014; 37: 152-158.

11 Mitchell GK, Tieman JJ, Shelby-James TM. Multidisciplinary care planning and teamwork in primary care. Med J Aust 2008; 188: S63.

12 Starfield B. Primary care is the health system's bedrock. BMJ 2013; 347: f4627.

13 Chokshi DA, Cohen L. Progress in primary care - from Alma-Ata to Astana. JAMA 2018; 320: 1965-1966.

14 Metersky ML, ZuWallack RL. Pulmonary rehabilitation for bronchiectasis: if not now, when? Eur Respir J 2019, 53: 1802474

15 Fox L, Onders R, Hermansen-Kobulnicky CJ, et al. Teaching interprofessional teamwork skills to health professional students: a scoping review. J Interprof Care 2018; 32: 127-135.

16 Nardini S, De Benedetto F, Sanguinetti CM, et al. COPD management as a model for all chronic respiratory conditions: report of the 4th Consensus Conference in Respiratory Medicine. Multidiscip Respir Med 2017; 10: 28 .

17 Hamad GA, Crooks M, Morice AH. The value of telehealth in the early detection of chronic obstructive pulmonary disease exacerbations: a prospective observational study. Health Informatics $J$ 2016; 22: 406-413.

18 Tsiligianni IG, van der Molen T, Tzanakis NE, et al. A telehealth integrated asthma-COPD service for primary care: a proposal for a pilot feasibility study in Crete, Greece. BMC Res Notes 2010; 3: 198

19 Wu G, Consunji M, Nelson RA, et al. Perspectives on managing solitary pulmonary nodules: a survey of primary care physicians. Semin Thorac Cardiovasc Surg 2017; 29: 391-405.

20 Haahtela T, Tuomisto LE, Pietinalho A, et al. A 10 year asthma programme in Finland: major change for the better. Thorax 2006; 61: 663-670.

21 Kinnula VL, Vasankari T, Kontula E, et al. The 10-year COPD programme in Finland: effects on quality of diagnosis, smoking, prevalence, hospital admissions and mortality. Prim Care Respir J 2011; 20: 178-183.

22 Sleurs K, Seys SF, Bousquet J, et al. Mobile health tools for the management of chronic respiratory diseases. Allergy 2019; 74: 1292-1306.

23 Cartwright M, Hirani SP, Rixon L, et al. Effect of telehealth on quality of life and psychological outcomes over 12 months (Whole Systems Demonstrator telehealth questionnaire study): nested study of patient reported outcomes in a pragmatic, cluster randomised controlled trial. BMJ 2013; 346: f653.

24 Henderson C, Knapp M, Fernández JL, et al. Cost effectiveness of telehealth for patients with long term conditions (Whole Systems Demonstrator telehealth questionnaire study): nested economic evaluation in a pragmatic, cluster randomised controlled trial. BMJ 2013; 346: f1035.

25 Cano I, Dueñas-Espín I, Hernandez C, et al. Protocol for regional implementation of community-based collaborative management of complex chronic patients. NPJ Prim Care Respir Med 2017; $27: 44$.

26 Vela E, Tényi Á, Cano I, et al. Population-based analysis of patients with COPD in Catalonia: a cohort study with implications for clinical management. BMJ Open 2018; 8: e017283.

27 Barberan-Garcia A, Ubré M, Roca J, et al. Personalised prehabilitation in high-risk patients undergoing elective major abdominal surgery: a randomized blinded controlled trial. Ann Surg 2018; 267: 50-56.

28 Barberan-Garcia A, Gimeno-Santos E, Blanco I, et al. Protocol for regional implementation of collaborative self-management services to promote physical activity. BMC Health Serv Res 2018; 18 : 560. 
29 Vargas C, Burgos F, Cano I, et al. Protocol for regional implementation of collaborative lung function testing. NPJ Prim Care Respir Med 2016; 26: 16024.

30 Gorini A, Mazzocco K, Triberti S, et al. A P5 Approach to m-Health: design suggestions for advanced mobile health technology. Front Psychol 2018; 9: 2066.

31 Knaul FM. Alleviating the abyss in palliative care and pain relief - an imperative of universal health coverage. Lancet 2018; 391: 1392-1454.

32 Vermylen JH, Szmuilowicz E, Kalhan R. Palliative care in COPD: an unmet area for quality improvement. Int J Chron Obstruct Pulmon Dis 2015; 10: 1543-1551.

33 Houben CHM, Spruit MA, Luyten $\mathrm{H}$, et al. Cluster-randomised trial of a nurse-led advance care planning session in patients with COPD and their loved ones. Thorax 2019; 74: 328-336.

34 Higginson IJ, Bausewein C, Reilly CC, et al. An integrated palliative and respiratory care service for patients with advanced disease and refractory breathlessness: a randomised controlled trial. Lancet Respir Med 2014; 2 P979-P987.

35 Smallwood $\mathrm{N}$, Thompson $\mathrm{M}$, Warrender-Sparkes $\mathrm{M}$, et al. Integrated respiratory and palliative care may improve outcomes in advanced lung disease. ERJ Open Res 2018; 4: 00102-2017.

36 Hjorth NE, Haugen DF, Schaufel MA. Advance care planning in life-threatening pulmonary disease: a focus group study. ERJ Open Res 2018; 4: 00101-2017.

37 Kreuter M, Bendstrup E, Russell A-M, et al. Palliative care in interstitial lung disease: living well. Lancet Respir Med 2017; 5: 968-980 